CRYSTALLOGRAPHIC COMMUNICATIONS

ISSN 2056-9890

Received 7 August 2015

Accepted 19 August 2015

Edited by H. Ishida, Okayama University, Japan

Keywords: crystal structure; polynaphthoxazine materials; oxazine; intramolecular hydrogen bond; $\mathrm{C}-\mathrm{H} \cdots \pi$ interactions; $\pi-\pi$ interaction

CCDC reference: 1419687

Supporting information: this article has supporting information at journals.iucr.org/e

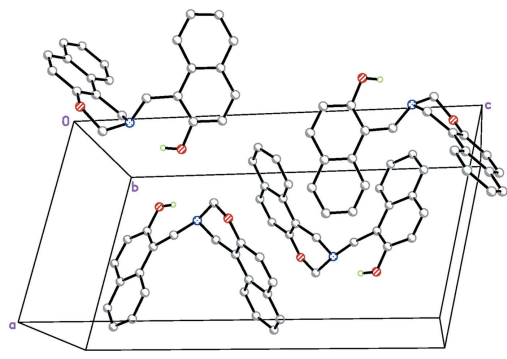

OPEN $\odot$ ACCESS

\section{Crystal structure of 1-[(2,3-dihydro-1H-naphtho- [1,2-e][1,3] oxazin-2-yl)methyl]naphthalen-2-ol: a possible candidate for new polynaphthoxazine materials}

\author{
Augusto Rivera, ${ }^{\mathrm{a} *}$ Jicli José Rojas, ${ }^{\mathrm{a}}$ Jaime Ríos-Motta ${ }^{\mathrm{a}}$ and Michael Bolte ${ }^{\mathrm{b}}$ \\ a Universidad Nacional de Colombia, Sede Bogotá, Facultad de Ciencias, Departamento de Química, Cra 30 No. 45-03, \\ Bogotá, Código Postal 111321, Colombia, and ${ }^{\mathbf{b}}$ Institut für Anorganische Chemie, J. W. Goethe-Universität Frankfurt, \\ Max-von Laue-Str. 7, 60438 Frankfurt/Main, Germany. *Correspondence e-mail: ariverau@unal.edu.co
}

In the title compound, $\mathrm{C}_{23} \mathrm{H}_{19} \mathrm{NO}_{2}$, an oxazine Mannich base derivative, the oxazine ring has a half-chair conformation. The 2-hydroxynaphthalen-1-yl substituent is placed in an axial position. There is an intramolecular $\mathrm{O}-\mathrm{H} \cdots \mathrm{N}$ hydrogen bond, forming an $S(6)$ graph-set motif. In the crystal, molecules are connected by a pair of $\mathrm{C}-\mathrm{H} \cdots \pi$ interactions into an inversion dimer, which is reinforced by another pair of weak $\mathrm{C}-\mathrm{H} \cdots \pi$ interactions. The dimers are linked by a $\pi-\pi$ interaction [centroid-centroid distance $=3.6268$ (17) $\AA$ ], consolidating a column along the $a$ axis. Furthermore, the columns interact with each other by a weak $\mathrm{C}-\mathrm{H} \cdots \pi$ interaction, generating a three-dimensional network.

\section{Chemical context}

Benzoxazines and naphthoxazines have been shown to polymerize via a thermally induced ring-opening reaction of the oxazine ring to form a phenolic structure associated with traditional phenolic resins (Ishida \& Sanders, 2001). Polybenzoxazines, polynaphthoxazines and their derivatives are a class of phenolic resins which are alternative to the traditional resins (Yildirim et al., 2006). So far the main contribution to the chemistry of these compounds has been the work of Burke (Burke, 1949; Burke et al., 1952), who was the first to show that aromatic oxazines could be obtained via Mannich-type condensation-cyclization reactions of certain phenols or naphtols with formaldehyde and primary amines in the molar ratio of 1:2:1. Various methods have been reported for the synthesis of dihydro-1,3-oxazines including the reaction under neat conditions via Mannich-type condensation-cyclization reaction of phenols or naphthols with formaldehyde and primary amines (Mathew et al., 2010). Our current research includes synthesis and characterization of monofunctional benzoxazines using aminals as performed Mannich electrophiles instead of formaldehyde and primary amines. Earlier (Rivera et al., 2005), we have reported an interesting behaviour of the macrocyclic aminal 1,3,6,8-tetraazatricyclo[4.4.1.1 ${ }^{3,8}$ ]dodecane (TATD) with hindered meta-disubstituted phenols affording 3,3-ethylene-bis(3,4-dihydro- $2 H$-1,3-benzoxazines) with good yields by a Mannich-type reaction in basic media. Recently, we synthesized the title compound by a reaction between the cyclic aminal 1,3,6,8-tetraazatricyclo[4.3.1.1. ${ }^{3,8}$ ] undecane (TATU) with 2-naphthol solventfree at low temperature. Because a wide range of cured properties can be obtained (Uyar et al., 2008) depending on the structure of aryloxazine monomers, initiators and the 
curing conditions, the title compound is a very good candidate as a monomer for the investigation of the polymerization of this class of compounds.

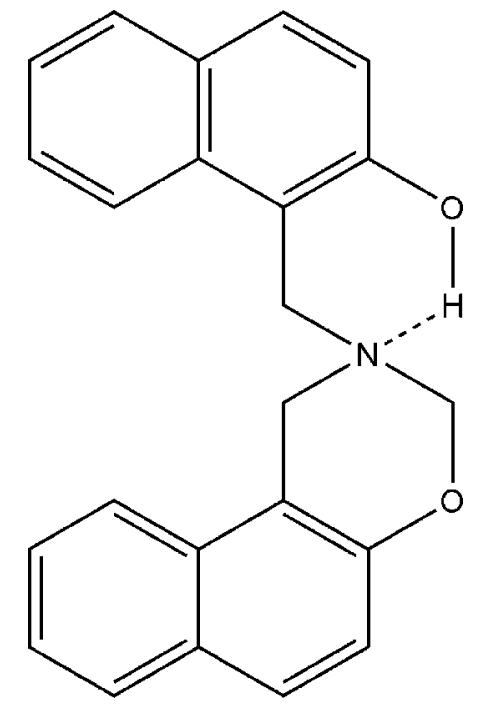

\section{Structural commentary}

The molecular structure of the title compound is shown in Fig. 1. The six-membered oxazine ring adopts a half-chair conformation with atoms $\mathrm{N} 1$ and $\mathrm{C} 1$ displaced by 0.323 (2) and 0.292 (3) A, respectively, from the mean plane composed of atoms $\mathrm{O} 1, \mathrm{C} 11, \mathrm{C} 12$ and $\mathrm{C} 2$. The puckering parameters are $Q=0.479$ (3) $\AA, \theta=50.0(3)^{\circ}$ and $\varphi=98.3$ (4) ${ }^{\circ}$ for the ring O1/ $\mathrm{C} 1 / \mathrm{N} 1 / \mathrm{C} 2 / \mathrm{C} 12 / \mathrm{C} 11$. The (2-hydroxynaphthalen-1-yl)methyl group bonded to atom N1 of the oxazine ring is placed in an axial position. The pendant naphthyl group (C21-C30) makes

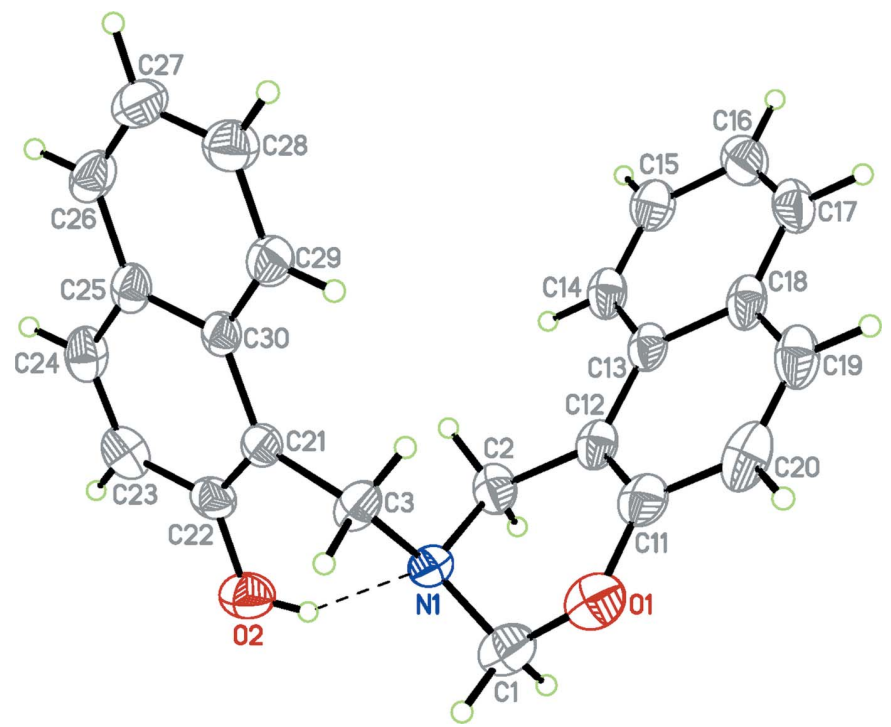

Figure 1

The molecular structure of the title compound, Displacement ellipsoids are drawn at the $50 \%$ probability level. The hydrogen bond is shown as a dashed line.
Table 1

Hydrogen-bond geometry $\left(\AA,^{\circ}\right)$.

$C g 2, C g 3$ and $C g 5$ are the centroids of the $\mathrm{C} 11-\mathrm{C} 13 / \mathrm{C} 18-\mathrm{C} 20, \mathrm{C} 13-\mathrm{C} 18$ and $\mathrm{C} 25-\mathrm{C} 30$ rings, respectively.

\begin{tabular}{lllll}
\hline$D-\mathrm{H} \cdots A$ & $D-\mathrm{H}$ & $\mathrm{H} \cdots A$ & $D \cdots A$ & $D-\mathrm{H} \cdots A$ \\
\hline $\mathrm{O} 2-\mathrm{H} 2 \cdots \mathrm{N} 1$ & 0.84 & 1.88 & $2.627(2)$ & 147 \\
$\mathrm{C} 1-\mathrm{H} 1 A \cdots C g 3^{\mathrm{i}}$ & 0.99 & 2.53 & $3.501(3)$ & 169 \\
$\mathrm{C} 2-\mathrm{H} 2 B \cdots C g 2^{\mathrm{i}}$ & 0.99 & 2.86 & $3.743(3)$ & 149 \\
$\mathrm{C} 14-\mathrm{H} 14 \cdots C g 5^{\mathrm{ii}}$ & 0.99 & 2.87 & $3.723(3)$ & 150 \\
\hline
\end{tabular}

Symmetry codes: (i) $-x+1,-y+1,-z+1$; (ii) $-x+\frac{1}{2}, y-\frac{1}{2},-z+\frac{3}{2}$.

a dihedral angle of $59.94(4)^{\circ}$ with the oxazine ring plane defined by atoms $\mathrm{C} 11, \mathrm{C} 12$ and $\mathrm{O} 1$. The bond lengths, $\mathrm{N} 1-\mathrm{C} 1$ and $\mathrm{O} 1-\mathrm{C} 1$, are normal and comparable to the corresponding values observed in the related structure of 6-bromo-2,4-bis(3methoxy-phenyl)-3,4-dihydro-2H-1,3-naphthoxazine (Sarojini et al., 2007). There is an intramolecular $\mathrm{O}-\mathrm{H} \cdots \mathrm{N}$ hydrogen bond (Table 1), forming an $S(6)$ graph-set motif, where the $\mathrm{N}$...O distance is longer by about 0.04 and $0.03 \AA$, respectively, than the observed values in related structures of 1-(piperidin-1-ylmethyl)-2-naphthol (Liu et al., 2005) and 1-morpholinomethyl-2-naphthol (Ma et al., 2005).

\section{Supramolecular features}

The crystal packing organization is essentially the result of two different types of interactions involving inversion-related molecules. Based on the distance criteria employed in PLATON (Spek, 2009), the most notable intermolecular contact is a $\mathrm{C}-\mathrm{H} \cdots \pi$ interaction $\left(\mathrm{C} 1-\mathrm{H} 1 A \cdots C g 3^{\mathrm{i}}\right.$; Table 1$)$, so that an inversion dimer is formed (Fig. 2). In addition, there is another $\mathrm{C}-\mathrm{H} \cdots \pi$ interaction $\left(\mathrm{C} 2-\mathrm{H} 2 B \cdots C g 2^{\mathrm{i}}\right.$; Table 1) in the dimer. A column of alternating inversion dimers extending along the $a$ axis results from a $\pi-\pi$ stacking interaction (Fig. 3) between adjacent 2,3-dihydro- $H$-naphtho[1,2-e][1,3]oxazine ring systems with a centroid-centroid distance of 3.6268 (17) $\AA$ [ $C g 3 \cdots C g 3^{\text {iii }}$; symmetry code: (iii) $=-x,-y+1$, $-z+1]$. Neighboring columns are connected by a weak $\mathrm{C}-$ $\mathrm{H} \cdots \pi$ interaction $\left(\mathrm{C} 14-\mathrm{H} 14 A \cdots C g 5^{\mathrm{ii}}\right.$; Table 1$)$, generating a

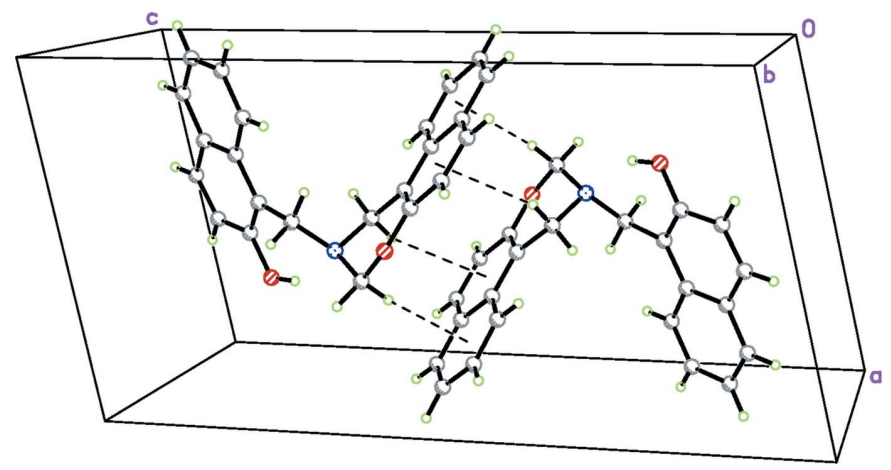

Figure 2

An inversion dimer in the crystal of the title compound, with $\mathrm{C}-\mathrm{H} \cdots \pi$ interactions indicated by dashed lines. 


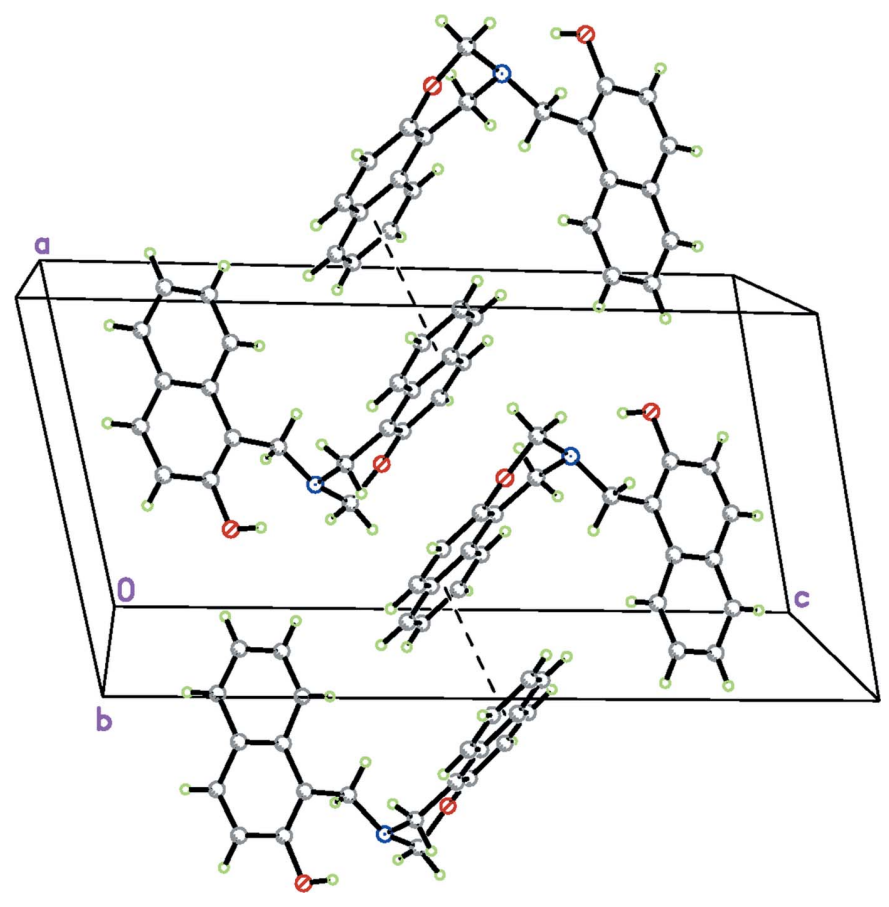

Figure 3

The view of the column structure along the $a$ axis, showing the $\pi-\pi$ stacking interactions (dashed lines).

three-dimensional network. The unit-cell packing is shown in Fig. 4.

\section{Database survey}

The 2,3-dihydro- $1 H$-naphtho[1,2-e][1,3]oxazine fragment is a quite rigid moiety. A search in the CSD (Groom \& Allen, 2014) for this fragment gave 22 hits with 24 fragments. The torsion angles in the heterocycle show broadly consistent values. Their absolute values are in the following ranges: $\mathrm{O}-$

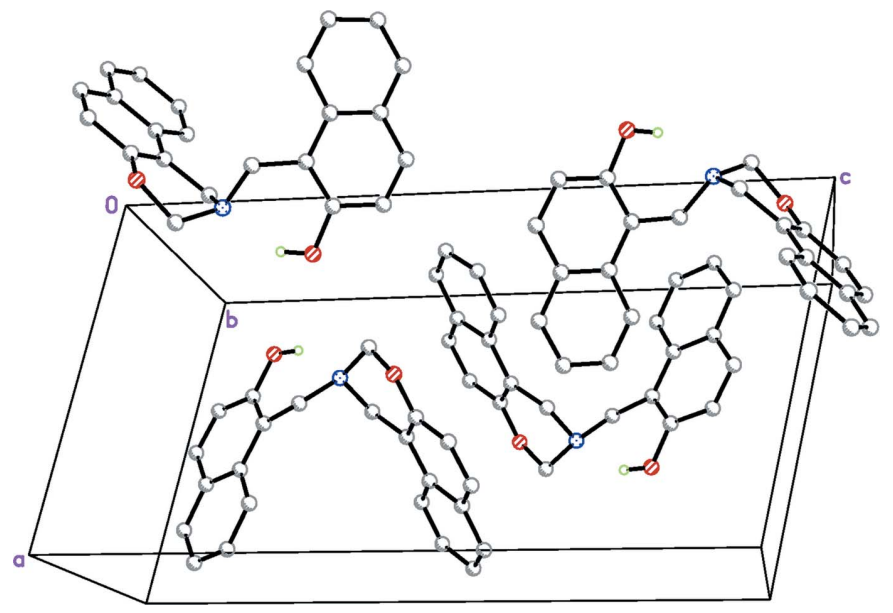

Figure 4

Packing diagram of the title compound. C-bound $\mathrm{H}$ atoms have been omitted for clarity.
Table 2

Experimental details.

\begin{tabular}{|c|c|}
\hline \multicolumn{2}{|l|}{ Crystal data } \\
\hline Chemical formula & $\mathrm{C}_{23} \mathrm{H}_{19} \mathrm{NO}_{2}$ \\
\hline$M_{\mathrm{r}}$ & 341.39 \\
\hline Crystal system, space group & Monoclinic, $P 2_{1} / n$ \\
\hline Temperature $(\mathrm{K})$ & 173 \\
\hline$a, b, c(\AA)$ & $9.6570(12), 9.7609$ (7), 18.790 (2) \\
\hline$\beta\left(^{\circ}\right)$ & $102.331(10)$ \\
\hline$V\left(\AA^{3}\right)$ & $1730.3(3)$ \\
\hline$Z$ & 4 \\
\hline Radiation type & Mo $K \alpha$ \\
\hline$\mu\left(\mathrm{mm}^{-1}\right)$ & 0.08 \\
\hline Crystal size $(\mathrm{mm})$ & $0.31 \times 0.11 \times 0.11$ \\
\hline \multicolumn{2}{|l|}{ Data collection } \\
\hline Diffractometer & STOE IPDS II two-circle \\
\hline Absorption correction & $\begin{array}{l}\text { Multi-scan }(X-A R E A ; \text { Stoe \& Cie } \\
\text { 2001) }\end{array}$ \\
\hline$T_{\min }, T_{\max }$ & $0.300,0.991$ \\
\hline $\begin{array}{l}\text { No. of measured, independent and } \\
\text { observed }[I>2 \sigma(I)] \text { reflections }\end{array}$ & $8807,3222,2069$ \\
\hline$R_{\text {int }}$ & 0.051 \\
\hline$(\sin \theta / \lambda)_{\max }\left(\AA^{-1}\right)$ & 0.608 \\
\hline \multicolumn{2}{|l|}{ Refinement } \\
\hline$R\left[F^{2}>2 \sigma\left(F^{2}\right)\right], w R\left(F^{2}\right), S$ & $0.053,0.135,0.96$ \\
\hline No. of reflections & 3222 \\
\hline No. of parameters & 237 \\
\hline $\mathrm{H}$-atom treatment & $\mathrm{H}$-atom parameters constrained \\
\hline$\Delta \rho_{\max }, \Delta \rho_{\min }\left(\mathrm{e} \AA^{-3}\right)$ & $0.57,-0.24$ \\
\hline
\end{tabular}

Computer programs: $X$-AREA (Stoe \& Cie, 2001), SHELXS97, XP in SHELXTL-Plus and SHELXL97 (Sheldrick, 2008), SHELXL2014/6 (Sheldrick, 2015).

$\mathrm{C}-\mathrm{N}-\mathrm{C} 56.0-69.7^{\circ}, \mathrm{C}-\mathrm{N}-\mathrm{C}-\mathrm{C} 37.7-53.8^{\circ}, \mathrm{N}-\mathrm{C}-\mathrm{C}-\mathrm{C}$ $3.7-24.2^{\circ}, \mathrm{C}-\mathrm{C}-\mathrm{C}-\mathrm{O} 0.1-6.3^{\circ}, \mathrm{C}-\mathrm{C}-\mathrm{O}-\mathrm{C} 1.0-21.6^{\circ}$ and $\mathrm{C}-\mathrm{O}-\mathrm{C}-\mathrm{N} 28.8-56.1^{\circ}$. Thus, it can be concluded that the conformation of this heterocycle is the same in all fragments. The values of the title compound fit very well into these ranges: $\mathrm{O} 1-\mathrm{C} 1-\mathrm{N} 1-\mathrm{C} 2 \quad 64.2(3)^{\circ}, \quad \mathrm{C} 1-\mathrm{N} 1-\mathrm{C} 2-$ $\mathrm{C} 12-48.9(3)^{\circ}, \mathrm{N} 1-\mathrm{C} 2-\mathrm{C} 12-\mathrm{C} 1119.3(3)^{\circ}, \mathrm{C} 2-\mathrm{C} 12-$ $\mathrm{C} 11-\mathrm{O} 1-0.5(3)^{\circ}, \mathrm{C} 12-\mathrm{C} 11-\mathrm{O} 1-\mathrm{C} 112.4(3)^{\circ}$ and $\mathrm{C} 11-$ $\mathrm{O} 1-\mathrm{C} 1-\mathrm{N} 1-45.3(3)^{\circ}$.

\section{Synthesis and crystallization}

2-Naphthol (144 mg, $1 \mathrm{mmol}$ ) and 1,3,6,8-tetraazatricyclo[4.3.1.1 $1^{3,8}$ undecane (TATU) (154 mg, $1 \mathrm{mmol}$ ) were manually ground together, heated to $313 \mathrm{~K}$ and stirred for $12 \mathrm{~h}$ under solvent-free conditions. Progress of the reaction was determined by TLC monitoring. After completion of the reaction, the mixture was cooled to room temperature and the solid residue was purified by silica gel column chromatography with benzene-ethyl acetate $(4: 1)$ as the eluent to give $1-\{[1 H$ naphtho[1,2-e][1,3]oxazin-2(3H)-yl]methyl\}naphthalen-2-ol as a brown solid in $28 \%$ yield. This compound was obtained in its crystalline form by recrystallization from an absolute ethanol solution (m.p. $443 \mathrm{~K}$ ).

\section{Refinement}

Crystal data, data collection and structure refinement details are summarized in Table 2. All $\mathrm{H}$ atoms were located in a 
difference electron-density map. The hydroxyl $\mathrm{H}$ atom was refined using a riding-model approximation with $\mathrm{O}-\mathrm{H}=$ $0.84 \AA$. The $U_{\text {iso }}(\mathrm{H})$ value and the $\mathrm{C}-\mathrm{C}-\mathrm{O}-\mathrm{H}$ torsion angle were refined. $\mathrm{C}$-bound $\mathrm{H}$ atoms were fixed geometrically $(\mathrm{C}-$ $\mathrm{H}=0.95$ or $0.99 \AA$ ) and treated as riding with $U_{\text {iso }}(\mathrm{H})=$ $1.2 U_{\mathrm{eq}}(\mathrm{C})$.

\section{Acknowledgements}

We acknowledge the Dirección de Investigaciones, Sede Bogotá (DIB) de la Universidad Nacional de Colombia, for financial support of this work (research project No. 28427). JJR thanks COLCIENCIAS for a fellowship.

\section{References}

Burke, W. J. (1949). J. Am. Chem. Soc. 71, 609-612.

Burke, W. J., Kolbezen, M. J. \& Stephens, C. W. (1952). J. Am. Chem. Soc. 74, 3601-3605.
Groom, C. R. \& Allen, F. H. (2014). Angew. Chem. Int. Ed. 53, 662671.

Ishida, H. \& Sanders, D. P. (2001). Polymer, 42, 3115-3125.

Liu, Q.-W., Zhang, M.-J., Wang, X.-Q. \& Ma, P.-G. (2005). Acta Cryst. E61, o4285-04286.

Ma, S.-S., Zhang, M.-J., Yuan, D.-Y. \& Qi, Z.-B. (2005). Acta Cryst. E61, o1370-o1371.

Mathew, B. P., Kumar, A., Sharma, S., Shukla, P. K. \& Nath, M. (2010). Eur. J. Med. Chem. 45, 1502-1507.

Rivera, A., Ríos, J., Quevedo, R. \& Joseph-Nathan, P. (2005). Rev. Colomb. Quim. 34, 105-115.

Sarojini, B. K., Narayana, B., Mayekar, A. N., Yathirajan, H. S. \& Bolte, M. (2007). Acta Cryst. E63, o4739.

Sheldrick, G. M. (2008). Acta Cryst. A64, 112-122.

Sheldrick, G. M. (2015). Acta Cryst. C71, 3-8.

Spek, A. L. (2009). Acta Cryst. D65, 148-155.

Stoe \& Cie (2001). X-AREA. Stoe \& Cie, Darmstadt, Germany.

Uyar, T., Koyuncu, Z., Ishida, H. \& Hacaloglu, J. (2008). Polym. Degrad. Stab. 93, 2096-2103.

Yildirim, A., Kiskan, B., Demirel, A. L. \& Yagci, Y. (2006). Eur. Polym. J. 42, 3006-3014. 


\section{supporting information}

Acta Cryst. (2015). E71, 1089-1092［https://doi.org/10.1107/S2056989015015583]

\section{Crystal structure of 1-[(2,3-dihydro-1H-naphtho[1,2-e] [1,3] oxazin-2-yl)methyl]-} naphthalen-2-ol: a possible candidate for new polynaphthoxazine materials

\section{Augusto Rivera, Jicli José Rojas, Jaime Ríos-Motta and Michael Bolte}

\section{Computing details}

Data collection: $X$-AREA (Stoe \& Cie, 2001); cell refinement: $X$-AREA (Stoe \& Cie, 2001); data reduction: $X$-AREA (Stoe \& Cie, 2001); program(s) used to solve structure: SHELXS97 (Sheldrick, 2008); program(s) used to refine structure:

SHELXL2014/6 (Sheldrick, 2015); molecular graphics: XP in SHELXTL-Plus (Sheldrick, 2008); software used to prepare material for publication: SHELXL97 (Sheldrick, 2008).

1-[(2,3-Dihydro-1H-naphtho[1,2-e][1,3] oxazin-2-yl)methyl]naphthalen-2-ol

\section{Crystal data}

$\mathrm{C}_{23} \mathrm{H}_{19} \mathrm{NO}_{2}$

$M_{r}=341.39$

Monoclinic, $P 2_{1} / n$

$a=9.6570(12) \AA$

$b=9.7609$ (7) $\AA$

$c=18.790(2) \AA$

$\beta=102.331(10)^{\circ}$

$V=1730.3(3) \AA^{3}$

$Z=4$

\section{Data collection}

STOE IPDS II two-circlediffractometer

Radiation source: Genix 3D I $\mu$ S microfocus Xray source

$\omega$ scans

Absorption correction: multi-scan

(X-AREA; Stoe \& Cie, 2001)

$T_{\min }=0.300, T_{\max }=0.991$

\section{Refinement}

Refinement on $F^{2}$

Least-squares matrix: full

$R\left[F^{2}>2 \sigma\left(F^{2}\right)\right]=0.053$

$w R\left(F^{2}\right)=0.135$

$S=0.96$

3222 reflections

237 parameters

0 restraints
$F(000)=720$

$D_{\mathrm{x}}=1.310 \mathrm{Mg} \mathrm{m}^{-3}$

Mo $K \alpha$ radiation, $\lambda=0.71073 \AA$

Cell parameters from 5838 reflections

$\theta=3.4-25.8^{\circ}$

$\mu=0.08 \mathrm{~mm}^{-1}$

$T=173 \mathrm{~K}$

Needle, colourless

$0.31 \times 0.11 \times 0.11 \mathrm{~mm}$

8807 measured reflections

3222 independent reflections

2069 reflections with $I>2 \sigma(I)$

$R_{\text {int }}=0.051$

$\theta_{\max }=25.6^{\circ}, \theta_{\min }=3.4^{\circ}$

$h=-9 \rightarrow 11$

$k=-10 \rightarrow 11$

$l=-22 \rightarrow 22$

Hydrogen site location: inferred from neighbouring sites

H-atom parameters constrained

$w=1 /\left[\sigma^{2}\left(F_{\mathrm{o}}^{2}\right)+(0.0738 P)^{2}\right]$

where $P=\left(F_{\mathrm{o}}{ }^{2}+2 F_{\mathrm{c}}{ }^{2}\right) / 3$

$(\Delta / \sigma)_{\max }<0.001$

$\Delta \rho_{\max }=0.57 \mathrm{e} \AA^{-3}$

$\Delta \rho_{\min }=-0.24$ e $\AA^{-3}$ 


\section{Special details}

Geometry. All e.s.d.'s (except the e.s.d. in the dihedral angle between two 1.s. planes) are estimated using the full covariance matrix. The cell e.s.d.'s are taken into account individually in the estimation of e.s.d.'s in distances, angles and torsion angles; correlations between e.s.d.'s in cell parameters are only used when they are defined by crystal symmetry. An approximate (isotropic) treatment of cell e.s.d.'s is used for estimating e.s.d.'s involving l.s. planes.

Fractional atomic coordinates and isotropic or equivalent isotropic displacement parameters $\left(\hat{A}^{2}\right)$

\begin{tabular}{|c|c|c|c|c|}
\hline & $x$ & $y$ & $z$ & $U_{\text {iso }} * / U_{\text {eq }}$ \\
\hline O1 & $0.5357(2)$ & $0.80192(19)$ & $0.57257(9)$ & $0.0422(5)$ \\
\hline $\mathrm{O} 2$ & $0.67542(18)$ & $0.5355(2)$ & $0.80171(9)$ & $0.0428(5)$ \\
\hline $\mathrm{H} 2$ & 0.6763 & 0.5678 & 0.7604 & $0.039(8)^{*}$ \\
\hline N1 & $0.5689(2)$ & $0.6438(2)$ & $0.67377(9)$ & $0.0332(5)$ \\
\hline $\mathrm{C} 1$ & $0.6360(3)$ & $0.7296(3)$ & $0.62925(13)$ & $0.0427(6)$ \\
\hline H1A & 0.6988 & 0.6725 & 0.6060 & $0.051^{*}$ \\
\hline $\mathrm{H} 1 \mathrm{~B}$ & 0.6961 & 0.7976 & 0.6607 & $0.051^{*}$ \\
\hline $\mathrm{C} 2$ & $0.4914(3)$ & $0.5345(3)$ & $0.62777(10)$ & $0.0336(6)$ \\
\hline $\mathrm{H} 2 \mathrm{~A}$ & 0.4305 & 0.4846 & 0.6553 & $0.040^{*}$ \\
\hline $\mathrm{H} 2 \mathrm{~B}$ & 0.5598 & 0.4684 & 0.6149 & $0.040^{*}$ \\
\hline $\mathrm{C} 3$ & $0.4775(3)$ & $0.7190(3)$ & $0.71422(11)$ & $0.0326(5)$ \\
\hline $\mathrm{H} 3 \mathrm{~A}$ & 0.3892 & 0.7470 & 0.6801 & $0.039 *$ \\
\hline $\mathrm{H} 3 \mathrm{~B}$ & 0.5269 & 0.8031 & 0.7356 & $0.039^{*}$ \\
\hline $\mathrm{C} 11$ & $0.4279(3)$ & $0.7211(3)$ & $0.53568(11)$ & $0.0346(6)$ \\
\hline $\mathrm{C} 12$ & $0.4002(2)$ & $0.5935(2)$ & $0.55868(10)$ & $0.0293(5)$ \\
\hline $\mathrm{C} 13$ & $0.2837(2)$ & $0.5172(3)$ & $0.51787(10)$ & $0.0292(5)$ \\
\hline $\mathrm{C} 14$ & $0.2464(3)$ & $0.3851(3)$ & $0.53810(10)$ & $0.0328(6)$ \\
\hline H14 & 0.3030 & 0.3424 & 0.5798 & $0.039^{*}$ \\
\hline $\mathrm{C} 15$ & $0.1310(3)$ & $0.3174(3)$ & $0.49924(11)$ & $0.0381(6)$ \\
\hline H15 & 0.1076 & 0.2294 & 0.5148 & $0.046^{*}$ \\
\hline $\mathrm{C} 16$ & $0.0471(3)$ & $0.3762(3)$ & $0.43679(11)$ & 0.0407 (6) \\
\hline H16 & -0.0330 & 0.3284 & 0.4100 & $0.049^{*}$ \\
\hline $\mathrm{C} 17$ & 0.0809 & $0.5027(3)$ & $0.41453(11)$ & $0.0388(7)$ \\
\hline H17 & 0.0246 & 0.5414 & 0.3716 & $0.047^{*}$ \\
\hline $\mathrm{C} 18$ & $0.1980(3)$ & $0.5774(3)$ & $0.45412(10)$ & $0.0336(6)$ \\
\hline C19 & $0.2309(3)$ & $0.7106(3)$ & $0.43329(11)$ & $0.0384(6)$ \\
\hline H19 & 0.1742 & 0.7510 & 0.3909 & $0.046^{*}$ \\
\hline $\mathrm{C} 20$ & $0.3419(3)$ & $0.7818(3)$ & $0.47263(11)$ & $0.0418(7)$ \\
\hline $\mathrm{H} 20$ & 0.3620 & 0.8716 & 0.4582 & $0.050^{*}$ \\
\hline $\mathrm{C} 21$ & $0.4405(2)$ & $0.6320(2)$ & $0.77453(10)$ & $0.0272(5)$ \\
\hline $\mathrm{C} 22$ & $0.5430(2)$ & $0.5495(3)$ & $0.81558(11)$ & $0.0311(5)$ \\
\hline $\mathrm{C} 23$ & $0.5180(3)$ & $0.4759(3)$ & $0.87625(11)$ & $0.0370(6)$ \\
\hline $\mathrm{H} 23$ & 0.5917 & 0.4229 & 0.9050 & $0.044 *$ \\
\hline $\mathrm{C} 24$ & $0.3891(3)$ & $0.4808(3)$ & $0.89361(11)$ & $0.0360(6)$ \\
\hline $\mathrm{H} 24$ & 0.3736 & 0.4314 & 0.9348 & $0.043^{*}$ \\
\hline $\mathrm{C} 25$ & $0.2767(3)$ & $0.5584(2)$ & $0.85145(10)$ & $0.0298(5)$ \\
\hline $\mathrm{C} 26$ & $0.1401(3)$ & $0.5588(3)$ & $0.86679(11)$ & $0.0365(6)$ \\
\hline $\mathrm{H} 26$ & 0.1231 & 0.5070 & 0.9069 & $0.044^{*}$ \\
\hline $\mathrm{C} 27$ & $0.0318(3)$ & $0.6318(3)$ & $0.82539(12)$ & $0.0406(6)$ \\
\hline
\end{tabular}


supporting information

\begin{tabular}{lllll}
$\mathrm{H} 27$ & -0.0603 & 0.6286 & 0.8357 & $0.049^{*}$ \\
$\mathrm{C} 28$ & $0.0574(3)$ & $0.7115(3)$ & $0.76759(12)$ & $0.0392(6)$ \\
$\mathrm{H} 28$ & -0.0175 & 0.7637 & 0.7392 & $0.047^{*}$ \\
$\mathrm{C} 29$ & $0.1886(2)$ & $0.7151(3)$ & $0.75153(10)$ & $0.0326(6)$ \\
$\mathrm{H} 29$ & 0.2037 & 0.7713 & 0.7126 & $0.039^{*}$ \\
$\mathrm{C} 30$ & $0.3031(2)$ & $0.6371(2)$ & $0.79162(9)$ & $0.0257(5)$ \\
\hline
\end{tabular}

Atomic displacement parameters $\left(\AA^{2}\right)$

\begin{tabular}{lllllll}
\hline & $U^{11}$ & $U^{22}$ & $U^{33}$ & $U^{12}$ & $U^{13}$ & $U^{23}$ \\
\hline $\mathrm{O} 1$ & $0.0455(11)$ & $0.0355(11)$ & $0.0494(9)$ & $-0.0013(9)$ & $0.0184(8)$ & $-0.0046(7)$ \\
$\mathrm{O} 2$ & $0.0317(9)$ & $0.0460(12)$ & $0.0510(10)$ & $0.0000(9)$ & $0.0094(7)$ & $-0.0033(8)$ \\
$\mathrm{N} 1$ & $0.0275(10)$ & $0.0433(13)$ & $0.0306(8)$ & $-0.0053(10)$ & $0.0104(7)$ & $-0.0037(8)$ \\
$\mathrm{C} 1$ & $0.0414(15)$ & $0.0423(17)$ & $0.0485(13)$ & $-0.0026(14)$ & $0.0192(11)$ & $-0.0015(11)$ \\
$\mathrm{C} 2$ & $0.0348(13)$ & $0.0365(15)$ & $0.0293(10)$ & $0.0095(12)$ & $0.0061(9)$ & $-0.0027(9)$ \\
$\mathrm{C} 3$ & $0.0407(14)$ & $0.0279(13)$ & $0.0334(10)$ & $-0.0053(12)$ & $0.0176(9)$ & $-0.0032(9)$ \\
$\mathrm{C} 11$ & $0.0358(13)$ & $0.0375(15)$ & $0.0345(10)$ & $0.0053(13)$ & $0.0164(9)$ & $-0.0074(10)$ \\
$\mathrm{C} 12$ & $0.0332(13)$ & $0.0302(14)$ & $0.0269(9)$ & $0.0099(11)$ & $0.0117(9)$ & $0.0005(8)$ \\
$\mathrm{C} 13$ & $0.0315(12)$ & $0.0341(14)$ & $0.0238(9)$ & $0.0110(11)$ & $0.0102(8)$ & $-0.0004(8)$ \\
$\mathrm{C} 14$ & $0.0400(14)$ & $0.0332(14)$ & $0.0263(9)$ & $0.0112(12)$ & $0.0095(9)$ & $0.0018(9)$ \\
$\mathrm{C} 15$ & $0.0448(15)$ & $0.0362(15)$ & $0.0349(11)$ & $0.0028(13)$ & $0.0123(10)$ & $-0.0023(10)$ \\
$\mathrm{C} 16$ & $0.0379(14)$ & $0.0501(18)$ & $0.0335(11)$ & $0.0070(14)$ & $0.0064(10)$ & $-0.0089(11)$ \\
$\mathrm{C} 17$ & $0.0374(14)$ & $0.0524(19)$ & $0.0260(10)$ & $0.0163(13)$ & $0.0052(9)$ & $-0.0009(10)$ \\
$\mathrm{C} 18$ & $0.0387(14)$ & $0.0398(15)$ & $0.0246(9)$ & $0.0166(12)$ & $0.0118(9)$ & $0.0034(9)$ \\
$\mathrm{C} 19$ & $0.0486(15)$ & $0.0387(15)$ & $0.0283(10)$ & $0.0165(14)$ & $0.0092(10)$ & $0.0070(10)$ \\
$\mathrm{C} 20$ & $0.0623(18)$ & $0.0313(15)$ & $0.0382(11)$ & $0.0129(14)$ & $0.0252(11)$ & $0.0062(10)$ \\
$\mathrm{C} 21$ & $0.0310(12)$ & $0.0256(12)$ & $0.0258(9)$ & $-0.0078(11)$ & $0.0077(8)$ & $-0.0066(8)$ \\
$\mathrm{C} 22$ & $0.0284(12)$ & $0.0302(14)$ & $0.0341(10)$ & $-0.0033(11)$ & $0.0052(9)$ & $-0.0087(9)$ \\
$\mathrm{C} 23$ & $0.0418(15)$ & $0.0288(14)$ & $0.0360(11)$ & $0.0030(12)$ & $-0.0014(10)$ & $-0.0002(9)$ \\
$\mathrm{C} 24$ & $0.0515(16)$ & $0.0279(13)$ & $0.0282(10)$ & $-0.0035(13)$ & $0.0077(10)$ & $0.0029(9)$ \\
$\mathrm{C} 25$ & $0.0405(14)$ & $0.0245(13)$ & $0.0257(9)$ & $-0.0071(11)$ & $0.0099(9)$ & $-0.0045(8)$ \\
$\mathrm{C} 26$ & $0.0453(15)$ & $0.0343(15)$ & $0.0349(11)$ & $-0.0123(13)$ & $0.0193(10)$ & $-0.0028(10)$ \\
$\mathrm{C} 27$ & $0.0354(14)$ & $0.0432(17)$ & $0.0475(12)$ & $-0.0084(13)$ & $0.0188(10)$ & $-0.0115(11)$ \\
$\mathrm{C} 28$ & $0.0335(13)$ & $0.0420(16)$ & $0.0416(11)$ & $0.0011(13)$ & $0.0070(10)$ & $-0.0017(11)$ \\
$\mathrm{C} 29$ & $0.0349(13)$ & $0.0338(14)$ & $0.0293(10)$ & $-0.0022(12)$ & $0.0075(9)$ & $0.0029(9)$ \\
$\mathrm{C} 30$ & $0.0308(12)$ & $0.0239(12)$ & $0.0228(8)$ & $-0.0061(10)$ & $0.0064(8)$ & $-0.0038(8)$ \\
& & & & & & \\
\hline & & & & & &
\end{tabular}

Geometric parameters $\left(\AA,{ }^{\circ}\right)$

\begin{tabular}{llll}
\hline $\mathrm{O} 1-\mathrm{C} 11$ & $1.370(3)$ & $\mathrm{C} 16-\mathrm{H} 16$ & 0.9500 \\
$\mathrm{O} 1-\mathrm{C} 1$ & $1.460(3)$ & $\mathrm{C} 17-\mathrm{C} 18$ & $1.416(4)$ \\
$\mathrm{O} 2-\mathrm{C} 22$ & $1.366(3)$ & $\mathrm{C} 17-\mathrm{H} 17$ & 0.9500 \\
$\mathrm{O} 2-\mathrm{H} 2$ & 0.8400 & $\mathrm{C} 18-\mathrm{C} 19$ & $1.413(4)$ \\
$\mathrm{N} 1-\mathrm{C} 1$ & $1.433(3)$ & $\mathrm{C} 19-\mathrm{C} 20$ & $1.357(4)$ \\
$\mathrm{N} 1-\mathrm{C} 2$ & $1.473(3)$ & $\mathrm{C} 19-\mathrm{H} 19$ & 0.9500 \\
$\mathrm{~N} 1-\mathrm{C} 3$ & $1.477(3)$ & $\mathrm{C} 20-\mathrm{H} 20$ & 0.9500 \\
$\mathrm{C} 1-\mathrm{H} 1 \mathrm{~A}$ & 0.9900 & $\mathrm{C} 21-\mathrm{C} 22$ & $1.377(3)$ \\
$\mathrm{C} 1-\mathrm{H} 1 \mathrm{~B}$ & 0.9900 & $\mathrm{C} 21-\mathrm{C} 30$ & $1.431(3)$
\end{tabular}




\begin{tabular}{|c|c|c|c|}
\hline $\mathrm{C} 2-\mathrm{C} 12$ & $1.518(3)$ & $\mathrm{C} 22-\mathrm{C} 23$ & $1.411(4)$ \\
\hline $\mathrm{C} 2-\mathrm{H} 2 \mathrm{~A}$ & 0.9900 & $\mathrm{C} 23-\mathrm{C} 24$ & $1.353(4)$ \\
\hline $\mathrm{C} 2-\mathrm{H} 2 \mathrm{~B}$ & 0.9900 & $\mathrm{C} 23-\mathrm{H} 23$ & 0.9500 \\
\hline $\mathrm{C} 3-\mathrm{C} 21$ & $1.518(3)$ & $\mathrm{C} 24-\mathrm{C} 25$ & $1.418(3)$ \\
\hline $\mathrm{C} 3-\mathrm{H} 3 \mathrm{~A}$ & 0.9900 & $\mathrm{C} 24-\mathrm{H} 24$ & 0.9500 \\
\hline $\mathrm{C} 3-\mathrm{H} 3 \mathrm{~B}$ & 0.9900 & $\mathrm{C} 25-\mathrm{C} 26$ & $1.409(3)$ \\
\hline $\mathrm{C} 11-\mathrm{C} 12$ & $1.363(4)$ & $\mathrm{C} 25-\mathrm{C} 30$ & $1.429(3)$ \\
\hline $\mathrm{C} 11-\mathrm{C} 20$ & $1.423(3)$ & $\mathrm{C} 26-\mathrm{C} 27$ & $1.364(4)$ \\
\hline $\mathrm{C} 12-\mathrm{C} 13$ & $1.429(3)$ & $\mathrm{C} 26-\mathrm{H} 26$ & 0.9500 \\
\hline $\mathrm{C} 13-\mathrm{C} 14$ & $1.412(4)$ & $\mathrm{C} 27-\mathrm{C} 28$ & $1.400(4)$ \\
\hline $\mathrm{C} 13-\mathrm{C} 18$ & $1.429(3)$ & $\mathrm{C} 27-\mathrm{H} 27$ & 0.9500 \\
\hline $\mathrm{C} 14-\mathrm{C} 15$ & $1.365(4)$ & $\mathrm{C} 28-\mathrm{C} 29$ & $1.364(3)$ \\
\hline C14-H14 & 0.9500 & $\mathrm{C} 28-\mathrm{H} 28$ & 0.9500 \\
\hline $\mathrm{C} 15-\mathrm{C} 16$ & $1.399(3)$ & $\mathrm{C} 29-\mathrm{C} 30$ & $1.420(3)$ \\
\hline $\mathrm{C} 15-\mathrm{H} 15$ & 0.9500 & $\mathrm{C} 29-\mathrm{H} 29$ & 0.9500 \\
\hline $\mathrm{C} 16-\mathrm{C} 17$ & $1.365(4)$ & & \\
\hline $\mathrm{C} 11-\mathrm{O} 1-\mathrm{C} 1$ & $113.9(2)$ & $\mathrm{C} 16-\mathrm{C} 17-\mathrm{H} 17$ & 119.3 \\
\hline $\mathrm{C} 22-\mathrm{O} 2-\mathrm{H} 2$ & 109.5 & $\mathrm{C} 18-\mathrm{C} 17-\mathrm{H} 17$ & 119.3 \\
\hline $\mathrm{C} 1-\mathrm{N} 1-\mathrm{C} 2$ & $108.51(17)$ & $\mathrm{C} 19-\mathrm{C} 18-\mathrm{C} 17$ & $122.0(2)$ \\
\hline $\mathrm{C} 1-\mathrm{N} 1-\mathrm{C} 3$ & $113.9(2)$ & $\mathrm{C} 19-\mathrm{C} 18-\mathrm{C} 13$ & $119.1(2)$ \\
\hline $\mathrm{C} 2-\mathrm{N} 1-\mathrm{C} 3$ & $112.13(18)$ & $\mathrm{C} 17-\mathrm{C} 18-\mathrm{C} 13$ & $118.9(2)$ \\
\hline $\mathrm{N} 1-\mathrm{C} 1-\mathrm{O} 1$ & $113.3(2)$ & $\mathrm{C} 20-\mathrm{C} 19-\mathrm{C} 18$ & $121.2(2)$ \\
\hline $\mathrm{N} 1-\mathrm{C} 1-\mathrm{H} 1 \mathrm{~A}$ & 108.9 & $\mathrm{C} 20-\mathrm{C} 19-\mathrm{H} 19$ & 119.4 \\
\hline $\mathrm{O} 1-\mathrm{C} 1-\mathrm{H} 1 \mathrm{~A}$ & 108.9 & $\mathrm{C} 18-\mathrm{C} 19-\mathrm{H} 19$ & 119.4 \\
\hline $\mathrm{N} 1-\mathrm{C} 1-\mathrm{H} 1 \mathrm{~B}$ & 108.9 & $\mathrm{C} 19-\mathrm{C} 20-\mathrm{C} 11$ & $119.5(3)$ \\
\hline $\mathrm{O} 1-\mathrm{C} 1-\mathrm{H} 1 \mathrm{~B}$ & 108.9 & $\mathrm{C} 19-\mathrm{C} 20-\mathrm{H} 20$ & 120.2 \\
\hline $\mathrm{H} 1 \mathrm{~A}-\mathrm{C} 1-\mathrm{H} 1 \mathrm{~B}$ & 107.7 & $\mathrm{C} 11-\mathrm{C} 20-\mathrm{H} 20$ & 120.2 \\
\hline $\mathrm{N} 1-\mathrm{C} 2-\mathrm{C} 12$ & $110.9(2)$ & $\mathrm{C} 22-\mathrm{C} 21-\mathrm{C} 30$ & $118.9(2)$ \\
\hline $\mathrm{N} 1-\mathrm{C} 2-\mathrm{H} 2 \mathrm{~A}$ & 109.5 & $\mathrm{C} 22-\mathrm{C} 21-\mathrm{C} 3$ & $119.3(2)$ \\
\hline $\mathrm{C} 12-\mathrm{C} 2-\mathrm{H} 2 \mathrm{~A}$ & 109.5 & $\mathrm{C} 30-\mathrm{C} 21-\mathrm{C} 3$ & $121.7(2)$ \\
\hline $\mathrm{N} 1-\mathrm{C} 2-\mathrm{H} 2 \mathrm{~B}$ & 109.5 & $\mathrm{O} 2-\mathrm{C} 22-\mathrm{C} 21$ & $122.8(2)$ \\
\hline $\mathrm{C} 12-\mathrm{C} 2-\mathrm{H} 2 \mathrm{~B}$ & 109.5 & $\mathrm{O} 2-\mathrm{C} 22-\mathrm{C} 23$ & $115.6(2)$ \\
\hline $\mathrm{H} 2 \mathrm{~A}-\mathrm{C} 2-\mathrm{H} 2 \mathrm{~B}$ & 108.1 & $\mathrm{C} 21-\mathrm{C} 22-\mathrm{C} 23$ & $121.6(2)$ \\
\hline $\mathrm{N} 1-\mathrm{C} 3-\mathrm{C} 21$ & $111.6(2)$ & $\mathrm{C} 24-\mathrm{C} 23-\mathrm{C} 22$ & $120.1(2)$ \\
\hline $\mathrm{N} 1-\mathrm{C} 3-\mathrm{H} 3 \mathrm{~A}$ & 109.3 & $\mathrm{C} 24-\mathrm{C} 23-\mathrm{H} 23$ & 119.9 \\
\hline $\mathrm{C} 21-\mathrm{C} 3-\mathrm{H} 3 \mathrm{~A}$ & 109.3 & $\mathrm{C} 22-\mathrm{C} 23-\mathrm{H} 23$ & 119.9 \\
\hline $\mathrm{N} 1-\mathrm{C} 3-\mathrm{H} 3 \mathrm{~B}$ & 109.3 & $\mathrm{C} 23-\mathrm{C} 24-\mathrm{C} 25$ & $121.2(2)$ \\
\hline $\mathrm{C} 21-\mathrm{C} 3-\mathrm{H} 3 \mathrm{~B}$ & 109.3 & $\mathrm{C} 23-\mathrm{C} 24-\mathrm{H} 24$ & 119.4 \\
\hline $\mathrm{H} 3 \mathrm{~A}-\mathrm{C} 3-\mathrm{H} 3 \mathrm{~B}$ & 108.0 & $\mathrm{C} 25-\mathrm{C} 24-\mathrm{H} 24$ & 119.4 \\
\hline $\mathrm{C} 12-\mathrm{C} 11-\mathrm{O} 1$ & $123.0(2)$ & $\mathrm{C} 26-\mathrm{C} 25-\mathrm{C} 24$ & $121.7(2)$ \\
\hline $\mathrm{C} 12-\mathrm{C} 11-\mathrm{C} 20$ & $121.7(2)$ & $\mathrm{C} 26-\mathrm{C} 25-\mathrm{C} 30$ & $119.5(2)$ \\
\hline $\mathrm{O} 1-\mathrm{C} 11-\mathrm{C} 20$ & $115.2(2)$ & $\mathrm{C} 24-\mathrm{C} 25-\mathrm{C} 30$ & $118.9(2)$ \\
\hline $\mathrm{C} 11-\mathrm{C} 12-\mathrm{C} 13$ & $119.45(19)$ & $\mathrm{C} 27-\mathrm{C} 26-\mathrm{C} 25$ & $121.5(2)$ \\
\hline $\mathrm{C} 11-\mathrm{C} 12-\mathrm{C} 2$ & $120.0(2)$ & $\mathrm{C} 27-\mathrm{C} 26-\mathrm{H} 26$ & 119.3 \\
\hline $\mathrm{C} 13-\mathrm{C} 12-\mathrm{C} 2$ & $120.5(2)$ & $\mathrm{C} 25-\mathrm{C} 26-\mathrm{H} 26$ & 119.3 \\
\hline $\mathrm{C} 14-\mathrm{C} 13-\mathrm{C} 12$ & $123.26(18)$ & $\mathrm{C} 26-\mathrm{C} 27-\mathrm{C} 28$ & $119.5(2)$ \\
\hline $\mathrm{C} 14-\mathrm{C} 13-\mathrm{C} 18$ & $117.8(2)$ & $\mathrm{C} 26-\mathrm{C} 27-\mathrm{H} 27$ & 120.3 \\
\hline
\end{tabular}




\begin{tabular}{|c|c|c|c|}
\hline $\mathrm{C} 12-\mathrm{C} 13-\mathrm{C} 18$ & $119.0(2)$ & $\mathrm{C} 28-\mathrm{C} 27-\mathrm{H} 27$ & 120.3 \\
\hline $\mathrm{C} 15-\mathrm{C} 14-\mathrm{C} 13$ & $121.6(2)$ & $\mathrm{C} 29-\mathrm{C} 28-\mathrm{C} 27$ & $120.8(2)$ \\
\hline $\mathrm{C} 15-\mathrm{C} 14-\mathrm{H} 14$ & 119.2 & $\mathrm{C} 29-\mathrm{C} 28-\mathrm{H} 28$ & 119.6 \\
\hline $\mathrm{C} 13-\mathrm{C} 14-\mathrm{H} 14$ & 119.2 & $\mathrm{C} 27-\mathrm{C} 28-\mathrm{H} 28$ & 119.6 \\
\hline $\mathrm{C} 14-\mathrm{C} 15-\mathrm{C} 16$ & $120.7(3)$ & $\mathrm{C} 28-\mathrm{C} 29-\mathrm{C} 30$ & $121.6(2)$ \\
\hline $\mathrm{C} 14-\mathrm{C} 15-\mathrm{H} 15$ & 119.7 & $\mathrm{C} 28-\mathrm{C} 29-\mathrm{H} 29$ & 119.2 \\
\hline $\mathrm{C} 16-\mathrm{C} 15-\mathrm{H} 15$ & 119.7 & $\mathrm{C} 30-\mathrm{C} 29-\mathrm{H} 29$ & 119.2 \\
\hline $\mathrm{C} 17-\mathrm{C} 16-\mathrm{C} 15$ & $119.6(2)$ & $\mathrm{C} 29-\mathrm{C} 30-\mathrm{C} 25$ & $117.2(2)$ \\
\hline $\mathrm{C} 17-\mathrm{C} 16-\mathrm{H} 16$ & 120.2 & $\mathrm{C} 29-\mathrm{C} 30-\mathrm{C} 21$ & $123.63(19)$ \\
\hline $\mathrm{C} 15-\mathrm{C} 16-\mathrm{H} 16$ & 120.2 & $\mathrm{C} 25-\mathrm{C} 30-\mathrm{C} 21$ & $119.2(2)$ \\
\hline $\mathrm{C} 16-\mathrm{C} 17-\mathrm{C} 18$ & $121.4(2)$ & & \\
\hline $\mathrm{C} 2-\mathrm{N} 1-\mathrm{C} 1-\mathrm{O} 1$ & $64.2(3)$ & $\mathrm{C} 13-\mathrm{C} 18-\mathrm{C} 19-\mathrm{C} 20$ & $0.0(4)$ \\
\hline $\mathrm{C} 3-\mathrm{N} 1-\mathrm{C} 1-\mathrm{O} 1$ & $-61.4(3)$ & $\mathrm{C} 18-\mathrm{C} 19-\mathrm{C} 20-\mathrm{C} 11$ & $0.6(4)$ \\
\hline $\mathrm{C} 11-\mathrm{O} 1-\mathrm{C} 1-\mathrm{N} 1$ & $-45.3(3)$ & $\mathrm{C} 12-\mathrm{C} 11-\mathrm{C} 20-\mathrm{C} 19$ & $-1.3(4)$ \\
\hline $\mathrm{C} 1-\mathrm{N} 1-\mathrm{C} 2-\mathrm{C} 12$ & $-48.9(3)$ & $\mathrm{O} 1-\mathrm{C} 11-\mathrm{C} 20-\mathrm{C} 19$ & $-178.8(2)$ \\
\hline $\mathrm{C} 3-\mathrm{N} 1-\mathrm{C} 2-\mathrm{C} 12$ & $77.7(2)$ & $\mathrm{N} 1-\mathrm{C} 3-\mathrm{C} 21-\mathrm{C} 22$ & $40.2(3)$ \\
\hline $\mathrm{C} 1-\mathrm{N} 1-\mathrm{C} 3-\mathrm{C} 21$ & $-165.05(18)$ & $\mathrm{N} 1-\mathrm{C} 3-\mathrm{C} 21-\mathrm{C} 30$ & $-142.0(2)$ \\
\hline $\mathrm{C} 2-\mathrm{N} 1-\mathrm{C} 3-\mathrm{C} 21$ & $71.3(2)$ & $\mathrm{C} 30-\mathrm{C} 21-\mathrm{C} 22-\mathrm{O} 2$ & $178.02(19)$ \\
\hline $\mathrm{C} 1-\mathrm{O} 1-\mathrm{C} 11-\mathrm{C} 12$ & $12.4(3)$ & $\mathrm{C} 3-\mathrm{C} 21-\mathrm{C} 22-\mathrm{O} 2$ & $-4.1(3)$ \\
\hline $\mathrm{C} 1-\mathrm{O} 1-\mathrm{C} 11-\mathrm{C} 20$ & $-170.1(2)$ & $\mathrm{C} 30-\mathrm{C} 21-\mathrm{C} 22-\mathrm{C} 23$ & $-3.5(3)$ \\
\hline $\mathrm{O} 1-\mathrm{C} 11-\mathrm{C} 12-\mathrm{C} 13$ & $178.6(2)$ & $\mathrm{C} 3-\mathrm{C} 21-\mathrm{C} 22-\mathrm{C} 23$ & $174.4(2)$ \\
\hline $\mathrm{C} 20-\mathrm{C} 11-\mathrm{C} 12-\mathrm{C} 13$ & $1.3(3)$ & $\mathrm{O} 2-\mathrm{C} 22-\mathrm{C} 23-\mathrm{C} 24$ & $-178.6(2)$ \\
\hline $\mathrm{O} 1-\mathrm{C} 11-\mathrm{C} 12-\mathrm{C} 2$ & $-0.5(3)$ & $\mathrm{C} 21-\mathrm{C} 22-\mathrm{C} 23-\mathrm{C} 24$ & $2.8(4)$ \\
\hline $\mathrm{C} 20-\mathrm{C} 11-\mathrm{C} 12-\mathrm{C} 2$ & $-177.8(2)$ & $\mathrm{C} 22-\mathrm{C} 23-\mathrm{C} 24-\mathrm{C} 25$ & $0.4(4)$ \\
\hline $\mathrm{N} 1-\mathrm{C} 2-\mathrm{C} 12-\mathrm{C} 11$ & $19.3(3)$ & $\mathrm{C} 23-\mathrm{C} 24-\mathrm{C} 25-\mathrm{C} 26$ & $176.7(2)$ \\
\hline $\mathrm{N} 1-\mathrm{C} 2-\mathrm{C} 12-\mathrm{C} 13$ & $-159.8(2)$ & $\mathrm{C} 23-\mathrm{C} 24-\mathrm{C} 25-\mathrm{C} 30$ & $-2.7(3)$ \\
\hline $\mathrm{C} 11-\mathrm{C} 12-\mathrm{C} 13-\mathrm{C} 14$ & $-179.3(2)$ & $\mathrm{C} 24-\mathrm{C} 25-\mathrm{C} 26-\mathrm{C} 27$ & $-178.9(2)$ \\
\hline $\mathrm{C} 2-\mathrm{C} 12-\mathrm{C} 13-\mathrm{C} 14$ & $-0.3(3)$ & $\mathrm{C} 30-\mathrm{C} 25-\mathrm{C} 26-\mathrm{C} 27$ & $0.5(3)$ \\
\hline $\mathrm{C} 11-\mathrm{C} 12-\mathrm{C} 13-\mathrm{C} 18$ & $-0.6(3)$ & $\mathrm{C} 25-\mathrm{C} 26-\mathrm{C} 27-\mathrm{C} 28$ & $-1.9(4)$ \\
\hline $\mathrm{C} 2-\mathrm{C} 12-\mathrm{C} 13-\mathrm{C} 18$ & $178.4(2)$ & $\mathrm{C} 26-\mathrm{C} 27-\mathrm{C} 28-\mathrm{C} 29$ & $1.1(4)$ \\
\hline $\mathrm{C} 12-\mathrm{C} 13-\mathrm{C} 14-\mathrm{C} 15$ & $177.5(2)$ & $\mathrm{C} 27-\mathrm{C} 28-\mathrm{C} 29-\mathrm{C} 30$ & $1.1(4)$ \\
\hline $\mathrm{C} 18-\mathrm{C} 13-\mathrm{C} 14-\mathrm{C} 15$ & $-1.2(3)$ & $\mathrm{C} 28-\mathrm{C} 29-\mathrm{C} 30-\mathrm{C} 25$ & $-2.4(3)$ \\
\hline $\mathrm{C} 13-\mathrm{C} 14-\mathrm{C} 15-\mathrm{C} 16$ & $1.3(4)$ & $\mathrm{C} 28-\mathrm{C} 29-\mathrm{C} 30-\mathrm{C} 21$ & $176.7(2)$ \\
\hline $\mathrm{C} 14-\mathrm{C} 15-\mathrm{C} 16-\mathrm{C} 17$ & $0.0(4)$ & $\mathrm{C} 26-\mathrm{C} 25-\mathrm{C} 30-\mathrm{C} 29$ & $1.6(3)$ \\
\hline $\mathrm{C} 15-\mathrm{C} 16-\mathrm{C} 17-\mathrm{C} 18$ & $-1.2(4)$ & $\mathrm{C} 24-\mathrm{C} 25-\mathrm{C} 30-\mathrm{C} 29$ & $-179.0(2)$ \\
\hline $\mathrm{C} 16-\mathrm{C} 17-\mathrm{C} 18-\mathrm{C} 19$ & $-177.5(2)$ & $\mathrm{C} 26-\mathrm{C} 25-\mathrm{C} 30-\mathrm{C} 21$ & $-177.5(2)$ \\
\hline $\mathrm{C} 16-\mathrm{C} 17-\mathrm{C} 18-\mathrm{C} 13$ & $1.2(4)$ & $\mathrm{C} 24-\mathrm{C} 25-\mathrm{C} 30-\mathrm{C} 21$ & $1.9(3)$ \\
\hline $\mathrm{C} 14-\mathrm{C} 13-\mathrm{C} 18-\mathrm{C} 19$ & $178.7(2)$ & $\mathrm{C} 22-\mathrm{C} 21-\mathrm{C} 30-\mathrm{C} 29$ & $-177.9(2)$ \\
\hline $\mathrm{C} 12-\mathrm{C} 13-\mathrm{C} 18-\mathrm{C} 19$ & $0.0(3)$ & $\mathrm{C} 3-\mathrm{C} 21-\mathrm{C} 30-\mathrm{C} 29$ & $4.3(3)$ \\
\hline $\mathrm{C} 14-\mathrm{C} 13-\mathrm{C} 18-\mathrm{C} 17$ & $0.0(3)$ & $\mathrm{C} 22-\mathrm{C} 21-\mathrm{C} 30-\mathrm{C} 25$ & $1.1(3)$ \\
\hline $\mathrm{C} 12-\mathrm{C} 13-\mathrm{C} 18-\mathrm{C} 17$ & $-178.8(2)$ & $\mathrm{C} 3-\mathrm{C} 21-\mathrm{C} 30-\mathrm{C} 25$ & $-176.70(19)$ \\
\hline $\mathrm{C} 17-\mathrm{C} 18-\mathrm{C} 19-\mathrm{C} 20$ & $178.7(2)$ & & \\
\hline
\end{tabular}




\section{supporting information}

Hydrogen-bond geometry $\left(A,{ }^{\circ}\right)$

$\mathrm{Cg} 2, \mathrm{Cg} 3$ and $\mathrm{Cg} 5$ are the centroids of the $\mathrm{C} 11-\mathrm{C} 13 / \mathrm{C} 18-\mathrm{C} 20, \mathrm{C} 13-\mathrm{C} 18$ and $\mathrm{C} 25-\mathrm{C} 30$ rings, respectively.

\begin{tabular}{lllll}
\hline$D-\mathrm{H} \cdots A$ & $D-\mathrm{H}$ & $\mathrm{H} \cdots A$ & $D \cdots A$ & $D-\mathrm{H} \cdots A$ \\
\hline $\mathrm{O} 2-\mathrm{H} 2 \cdots \mathrm{N} 1$ & 0.84 & 1.88 & $2.627(2)$ & 147 \\
$\mathrm{C} 1-\mathrm{H} 1 A \cdots C g 3^{\mathrm{i}}$ & 0.99 & 2.53 & $3.501(3)$ & 169 \\
$\mathrm{C} 2-\mathrm{H} 2 B \cdots C g 2^{\mathrm{i}}$ & 0.99 & 2.86 & $3.743(3)$ & 149 \\
$\mathrm{C} 14-\mathrm{H} 14 \cdots C g 5^{\mathrm{ii}}$ & 0.99 & 2.87 & $3.723(3)$ & 150 \\
\hline
\end{tabular}

Symmetry codes: (i) $-x+1,-y+1,-z+1$; (ii) $-x+1 / 2, y-1 / 2,-z+3 / 2$. 